\title{
Conduite des essais cliniques de médicaments au niveau européen depuis la directive européenne essais cliniques. Revue des aspects réglementaires et pratiques dans différents pays
}

\author{
1 Pharmacologie Clinique, CHU Clermont Ferrand, Clermont Ferrand, France \\ 2 IRIS, Groupe Servier, Courbevoie, France \\ 3 Spriet Conseil, Paris, France
}

Claude Dubray ${ }^{1}$, Patricia Maillere ${ }^{2}$, Alain Spriet ${ }^{3}$ et les participants de la table ronde $n^{\circ} 1$ de Giens XXII*

\section{Mots clés :}

Comité de Protection des Personnes (CPP) essai clinique ; pharmacovigilance ; Afssaps

\begin{abstract}
Résumé - La directive essais cliniques avait pour but une harmonisation des législations afin de favoriser la cohérence du système européen et par la même l'attractivité de l'Europe en matière de recherche clinique tout en maintenant ou renforçant la protection des personnes qui acceptent d'y participer.

La situation française en matière administrative était jusqu'à présent relativement favorable (étude à notifier avec déjà un seul comité d'éthique par étude), il est donc important de maintenir la compétitivité sur ces points sachant que sur d'autres points (délais de mise en place des conventions, recrutement, ...) la situation n'est pas à l'avantage de la France.

À l'heure actuelle, la transposition de cette directive n'est pas encore totalement finalisée en droit français. La période pilote qui a été mise en place par l'Afssaps (Agence française de sécurité sanitaire des produits de santé) a permis d'établir sans délais les modalités de mise en place.

Pour les CPP (Comités de Protection des Personnes), la situation est plus critique dans la mesure où beaucoup de points restent à établir (choix des membres, règlement intérieur, rapport $\mathrm{CA} / \mathrm{CE}$ [autorités compétentes/comités d'éthique]...). L'atelier de Giens a permis de faire naître un certain nombre de propositions et souhaite par le biais du groupe de pilotage Afssaps / DGS (Direction Générale de la Santé) / LEEM (Les Entreprises du Médicament) continuer à contribuer à la mise en place d'un système efficace et protecteur pour les patients.
\end{abstract}

\section{Introduction}

Chaque pays européen a mis en place de manière indépendante sa propre législation en matière d'essais cliniques. Certains pays demandaient une autorisation préalable des autorités de santé pour tout essai, d'autres ne l'exigeaient qu'à partir des essais chez le patient, d'autres demandaient une notification. Certains pays avaient mis en place un processus d'accord par le biais d'un seul comité d'éthique par étude, d'autres exigeaient l'accord d'un comité par centre. Les éléments nécessaires à la soumission étaient différents d'un pays à l'autre, ainsi que les demandes en matière de mise à jour et d'information en cours d'essai.

\footnotetext{
* Pour la liste des participants, voir en fin d'article.
}

À partir des années 90, la Commission européenne a émis le souhait d'une harmonisation des législations afin de favoriser la cohérence du système européen et par la même l'attractivité de l'Europe en matière de recherche clinique tout en maintenant ou renforçant la protection des personnes qui acceptent d'y participer.

La directive européenne a été publiée en version finale le 28 novembre 2001 et la transposition dans les différents pays est en cours d'achèvement. Lors des travaux de la table-ronde, un certain nombre de décrets d'application étaient encore manquants notamment ceux concernant le fonctionnement des comités d'éthiques.

Les différentes enquêtes menées notamment par le LEEM ont montré que, par le passé, le système administratif français était relativement clément dans la mesure où les études donnaient lieu à notification et pas à autorisation et que la loi Huriet avait 
permis l'instauration d'un système simple avec une autorisation d'un CCP par étude.

L'objectif défini était d'examiner la situation française par rapport à celles des autres principaux pays européens et d'élaborer des propositions pour maintenir ou améliorer son attractivité.

Les réflexions ont été menées en parallèle dans le cadre de 5 sous-groupes :

- états des lieux;

- les Comités de Protection des Personnes;

- les conditions financières;

- la pharmacovigilance;

- les registres des essais cliniques.

\section{2. États des lieux}

Ce sous-groupe «État des lieux » était animé par Christine Marey et a élaboré des tableaux comparant les modalités de transposition de la directive dans 10 pays : Allemagne, Danemark, Espagne, Finlande, France, Italie, Norvège, Pologne, République tchèque et Suède sur la base des textes existants sans tenir compte d'autres dispositions réglementaires éventuelles ni des habitudes culturelles, mais en tenant compte de la pratique des participants au sous-groupe.

De nombreux items ont été étudiés : rôle et responsabilité des autorités compétentes et comité d'éthique, composition, procédures administratives, autorisation de lieux, registres, compensations...

Des tableaux sont disponibles sur le site des ateliers de Giens. $^{[1]}$

Concrètement, pendant la phase pilote pour l'Afssaps, alors que les évaluations par les comités d'éthique étaient encore régies par la loi Huriet, la France a gardé des délais compétitifs en matière d'autorisation des essais. Le délai d'inclusion du premier patient est en moyenne de 110 à 115 jours, le point crucial étant souvent le délai de négociation du contrat avec l'hôpital.

La première recommandation du groupe adressée aux directeurs d'hôpitaux est donc d'accepter de commencer la préparation des conventions hospitalières sans attendre l'accord du CCP, ce qui est encore souvent le cas.

En matière de délai, l'Afssaps se positionne de façon compétitive en incluant la période de validation dans le délai de 60 jours, sans prévoir de clock-stop en cas de questions. Si l'industriel ne peut répondre dans le délai imparti, le dossier doit être redéposé. La deuxième recommandation du groupe est dans ce cas de ne pas demander que les redevances soient de nouveau payées pour éviter toute perte de temps inutile. En matière de transparence du délai, il faut noter que l'Afssaps donne un accusé de réception avec la date du J60. Les efforts déjà notés pour rendre les évaluateurs internes plus accessibles pour tout éclaircissement demandé par les promoteurs doivent être encouragés. C'est le cas pour des pays majeurs (Grande Bretagne, Suède, ...) et permet réellement d'accélérer la fin de procédure.

Un certain nombre d'idées en provenance d'autres pays pourraient être mises en place en France, comme par exemple : (i) procédures d'appel comme il en existe en Grande Bretagne, tant au niveau de l'autorité compétente que du comité d'éthique; (ii) la notion de «co-sponsor» : les responsabilités en matière de gestion du dossier d'autorisation d'études, de conduite de l'essai ou de gestion de la pharmacovigilance peuvent être attribuées à des entités différentes; (iii) accord avec remarques (pratiqué par la Grande Bretagne, l'Allemagne, la Suède, la Finlande, la Pologne).

La fourniture des médicaments expérimentaux a fait l'objet d'une discussion particulière car c'est un frein important dans les recherches académiques. Actuellement, la recherche non commerciale est 5 fois plus importante aux USA (États-Unis) qu'en Europe et ce rapporté au nombre d'habitants. Un projet de « guideline » européen prévoit une aide possible de l'industriel sans qu'il ne soit promoteur. Si l'on veut que cela puisse se faire en réalité, il faut que celui-ci ne risque pas d'être requalifié promoteur en cas de problème autre que celui dû à un problème de qualité au niveau des produits.

Certains pays européens dont la France prévoient la possibilité d'une prise en charge par les organismes de santé avec des modalités différentes en fonction des systèmes. En France, les caisses peuvent prendre en charge à titre dérogatoire les médicaments faisant l'objet d'une recherche biomédicale si le produit est utilisé dans son indication remboursée ou si le ministre le décide après avis de l'UNCAM (Union Nationale des Caisses d'Assurance Maladie) et de la HAS (Haute Autorité de Santé) sur un critère d'intérêt de santé publique.

Il est important que cette opportunité existe. Malgré tout, le délai nécessaire à cette procédure reste un obstacle.

Une solution plus rapide pourrait venir du texte actuellement en discussion au Parlement et qui prévoit d'inclure le médicament dans les études sur les soins courants. Le sujet reste à suivre de manière précise.

\section{Les CPP}

Ce deuxième sous-groupe a été animé par Philippe Rusch.

La directive européenne prévoit un unique accord de comité d'éthique par étude. En réalité, dans beaucoup de pays, l'accord se fait toujours par le comité de chaque hôpital (ex Grande Bretagne) ou province (Allemagne ou Espagne). La France, en matière de simplification de procédure, se positionne donc toujours de façon favorable. 
En matière de responsabilité, les deux structures Afssaps et CPP évaluent la méthodologie de l'essai avec donc le risque d'aboutir à des avis divergents. Ce cas de figure devra être évalué. Il est clair que l'Afssaps a forcément une vision plus globale puisqu'elle évaluera tous les protocoles liés à un même produit, à tous les produits de la même classe. Les membres des CPP présents ont indiqué qu'il était toujours utile de fournir dans le dossier une évaluation méthodologique indépendante si elle est disponible, exemple : cas des «scientific advice» européens.

Dans le cadre de la transposition de la directive, beaucoup de décrets manquent encore et tous les CPP n'ont pas été renommés.

Les points forts du système français ont été identifiés : (i) le nombre de CPP sera le même que les anciens CCPPRB (comité consultatif de protection des personnes dans la recherche biomédicale), l'inter-régionalité doit permettre une gestion rapide des dossiers; (ii) un certain nombre de points faibles sont à noter; (iii) 10 à $40 \%$ des membres doivent encore être identifiés ; (iv) la gestion des anciens dossiers et de leurs amendements doit être définie de manière pragmatique; (v) la légalité des avis doit être bien étudiée : ils sont maintenant contraignants alors qu'ils n'étaient auparavant que consultatifs. Il faut donc définir un format qui permette de confirmer la validité de l'avis (quorum... ); (vi) le Décret sur les collections est très attendu. Plusieurs propositions ont été faites : avis favorable par défaut, définition de la fin de recherche de manière pragmatique en fonction du protocole, travail sur la collection et changement de finalité.

Beaucoup de sujets restent à clarifier afin que la performance des CPP ne soit pas affectée : fonctionnement, dialogue Afssaps/CPP, règlement intérieur.

Plusieurs recommandations ont été faites pour conserver l'acquis compétitif au niveau des CPP :

- la France a choisi d'inclure dans les CPP des membres des associations de patients. Ces postes ont été pourvus dans pratiquement tous les CPP. Leur formation est un processus à consolider. À noter 160 membres ont été formés par EurordisInserm. Le sujet de la formation à l'évaluation se pose pour tout nouveau membre;

- le décret d'avril 2006 demande la présence obligatoire d'un bio-statisticien. En pratique, ce point s'avère très problématique et la validité des accords de CPP risque d'être mise à mal. Il est donc proposé de modifier le décret afin de remplacer le bio-statisticien par une personne compétente en méthodologie.

Le sujet de l'harmonisation des avis des comités d'éthique à l'échelon européen a été évoqué dans le cadre notamment du Réseau européen : EUREC (European Network of Research Ethics Committees $)^{[2]}$ qui regroupe les comités des pays suivants :
Grande Bretagne, Danemark, Allemagne, Espagne, Italie, France, Suisse, Finlande, Lituanie, Slovaquie.

Les premiers travaux de ce groupe portent sur la description des dispositifs existants, les modalités de travail, et la définition d'un corpus utile à la formation dans tous les pays. Des différences importantes ont été identifiées très vite, comme par exemple : (i) certains comités se fixent comme objectif l'évaluation méthodologique de l'essai, d'autres souhaitent se focaliser sur la protection des personnes; (ii) certains souhaitent former leurs membres afin qu'ils soient réellement des professionnels de l'évaluation d'autres souhaitent impérativement garder la naïveté des représentants de la société civile.

Ce dialogue, même difficile, mérite d'être encouragé.

\section{Registres}

Le troisième sous-groupe animé par Claire Sibenaler a étudié spécifiquement le sujet des registres dans la mesure où l'Afssaps prévoit la mise en place d'un registre.

De très nombreux registres existent maintenant.

Tout d'abord, afin d'améliorer la transparence sur le montage des essais, les grands journaux ont demandé à ce que chaque essai pour être publié soit listé sur un site indépendant du promoteur. Concrètement, actuellement seuls deux sites sont agréés. ${ }^{[3,4]}$

Les industriels ont indiqué qu'ils mettraient en ligne, souvent sur leurs propres sites, les protocoles et les résultats de leurs propres essais. Quelques points restent à discuter en matière de délai : les études de preuves, de concept et quelques critères secondaires qui peuvent apporter des éléments confidentiels pour un futur développement. Le portail de la FIIM (IFPMA International Federation of Pharmaceutical Manufacturers \& Associations) ${ }^{[5]}$ existe et facilite les requêtes, l'OMS (Organisation Mondiale de la Santé) annonce aussi la mise en place d'un portail. Des sites institutionnels sont en projet : Europharm doit mettre en ligne des informations sur les produits sur le marché (Résumés des Caractéristiques, Notices...), et annoncer les protocoles des études en cours.

Tout le monde s'accorde à dire que la multiplication de ces bases d'information n'est pas souhaitable, il sera extrêmement difficile de les mettre à jour et d'éviter les informations divergentes.

En France, la base de l'Afssaps existe déjà pour certaines pathologies (maladies rares, hépatites), l'INCA (Institut national du Cancer) souhaite faire de même pour les essais dans le cancer.

Quelques agences à l'échelon européen souhaitent comme l'Afssaps mettre un site d'essais cliniques en ligne (Espagne, Italie). L'intérêt spécifique de la base de l'Afssaps est de fournir une information en français pour le patient français. Il est proposé de ne pas faire de transfert automatique des champs 
d'EudraCT (problème de compréhension) et de pas livrer d'informations considérées encore confidentielles par les promoteurs (industriels ou académiques).

\section{Pharmacovigilance}

Le quatrième sous-groupe animé par Véronique Lamarque a traité des notifications de Pharmacovigilance.

Le sujet de fond est celui de la quantité d'information délivrée conformément à la réglementation et ce qui est réellement utile de retenir et d'évaluer.

Les autorités compétentes des différents pays ont déjà l'habitude de recevoir les notifications spontanées en cours d'essais, ceci est nouveau pour les CPP qui doivent maintenant définir quelle est leur mission par rapport à ces informations, comment les gérer et les évaluer. .

Les recommandation suivantes ont été proposées :

- utilisation généralisée du formulaire CIOMS (Council for Intenational Organizations of Medical Sciences), référence claire à l'essai, info limitée aux SUSAR (SUspected Severe Adverse Reactions) réellement, tenant compte des événements prédéfinis dans le protocole;

- rapports de synthèse périodiques aboutissant à des conclusions claires et digérées ;

- revoir au niveau européen l'obligation des envois des cas individuels aux comités d'éthique;

- étudier au niveau européen la possibilité de définir un responsable spécifique de la gestion de la Pharmacovigilance pour une structure dans le cadre de la co-promotion;

- suivre attentivement le texte sur la Vigilance des Médicaments Non Expérimentaux qui est en cours d'élaboration.

\section{Conditions financières}

Le dernier sous-groupe animé par Elisabeth Rouffiac a recherché des différences en matières de coûts et d'indemnisation d'un pays à l'autre.

Les frais d'évaluation demandés par les autorités compétentes ou les comités d'éthique (dossier d'autorisation, inspections, évaluation des rapports annuels...) ne sont pas actuellement des éléments discriminants dans le choix des pays. Des montants exceptionnels ont été évoqués pour certains comités en dehors de la France, ce point reste donc à surveiller.. .

Les honoraires des intervenants de la recherche posent des problèmes particuliers en France. Ces informations sont classiquement évaluées par les comités d'éthique en dehors de France et portent peu à discussion. Les montants sont en France évalués par le CNO (Conseil National de l'Ordre), ce peut être une source de souci en matière de délai dans la mise en place de l'essai. Le dialogue Promoteurs/CNO doit se poursuivre sur ce sujet.

L'indemnisation des patients est désormais possible en France comme dans d'autres pays (Grande Bretagne, Allemagne, Espagne...). Seules les contraintes subies peuvent être dédommagées, son principe et les montants sont au préalable évaluées par les CPP.

\section{Conclusion}

La directive essais cliniques avait pour but une harmonisation des législations afin de favoriser la cohérence du système européen et par la même l'attractivité de l'Europe en matière de recherche clinique tout en maintenant ou renforçant la protection des personnes qui acceptent d'y participer.

La situation française en matière administrative était jusqu'à présent relativement favorable (étude à notifier avec déjà un seul comité d'éthique par étude), il est donc important de maintenir la compétitivité sur ces points sachant que sur d'autres points (délais de mise en place des conventions, recrutement, ...) la situation n'est pas à l'avantage de la France.

À l'heure actuelle, la transposition de cette directive n'est pas encore totalement finalisée en droit français. La période pilote qui a été mise en place par l'Afssaps a permis d'établir sans délais les modalités de mise en place.

Pour les CPP, la situation est plus critique dans la mesure où beaucoup de points restent à établir (choix des membres, règlement intérieur, rapport CA CE, ... ). L'atelier de Giens a permis de faire naître un certain nombre de propositions et souhaite par le biais du groupe de pilotage Afssaps/DGS/LEEM continuer à contribuer à la mise en place d'un système efficace et protecteur pour les patients.

\section{Participants}

Michel Abitboul (Quintiles), Béatrice Barraud (INSERMDAPS), Marie Castera-Tellier (CHU, Lille), François Chapuis (CHU, Lyon), François Faurisson (Eurordis), Elizabeth FrijaOrvoen (Présidente CPP Hotel Dieu, Paris), Jean Genève (FNCLCC), Anne-Sophie Ginon (MCF en droit Université Paris X, Nanterre), Corinne Guérin (Groupe Hospitalier Cochin), Thierry Herguetta (Président CPP Salpétrière, Paris), Denis Lacombe (EORTC), Véronique Lamarque (Pfizer), Christian Libersa (Pharmacologie, Faculté de Médecine, Lille), Brigitte Marchenay (Roche), Christine Marey (Servier), Annie Métro (ANRS), Armelle Mijonnet (Merck Sharp \& Dohme Chibret), Claire Orefice (Cythéris), Gérard Pons (Hôpital St Vincent de Paul, 
Paris), Nathalie Quideau (Sanofi), Isabelle Richard-Lordereau (Glaxo Smith Kline), Christian Rollin (Bristol Myers Squibb), Elisabeth Rouffiac (Abbott France/Pharmacie), Philippe Rusch (Président CPPRB, Saint-Etienne), Claire Sibenaler (LEEM), Sandra Touratier (Afssaps, Unité Essais Cliniques).

\section{Références}

1. http://wWw.ateliersdegiens.org
2. http://www. eurecnet.org

3. http://www.clinicaltrials.gov

4. http://www. controlled-trials.com

5. http://www.ifpma.org

Correspondance et offprints : Patricia Maillere, IRIS, Groupe Servier, 6 place des Pléiades, 92415 Courbevoie Cedex, France.

E-mail : maillere@fr.netgrs.com 